

В. Н. Дубинин, Принцип мажорации для $p$-листных функций, Матем. заметки, 1999, том 65, выпуск 4, 533-541

DOI: https://doi.org/10.4213/mzm1079

Использование Общероссийского математического портала Math-Net.Ru подразумевает, что вы прочитали и согласны с пользовательским соглашением http://www.mathnet.ru/rus/agreement

Параметры загрузки:

IP: 3.93 .64 .190

26 апреля 2023 г., 14:37:31

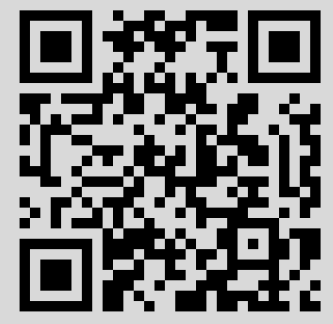




\section{ПРИНЦИП МАЖОРАЦИИ ДЛЯ $p$-ЛИСТНЫХ ФУНКЦИЙ}

\section{В.Н. Дубинин}

Доказана теорема, дополняющая известньй принцип Линделёфа для функций Грина и неравенство Митюка в случае $p$-листных отображений. Приведены приложения этой теоремы и метода симметризации в геометрической теории функций комплексного переменного.

Библиограффия: 9 названий.

1. Введение. Пусть $B$ и $D$ - области, имеющие функции $Г$ рина $g_{B}\left(z, z^{\prime}\right)$ и $g_{D}\left(w, w^{\prime}\right)$ и расположенные в открытых плоскостях $\mathbb{C}_{z}$ и $\mathbb{C}_{w}$ соответственно. Пусть $w=f(z)-$ регулярная функция в $B$, значения которой лежат в $D$. Одним из основных принципов мажорации в теории конформного отображения является

ПРИНЦИП ЛИНДЕЛЁФА. Если $w_{0}$ - точка образа $f(B)$ области $B ; z_{0}, z_{1}, z_{2}, \ldots$ -

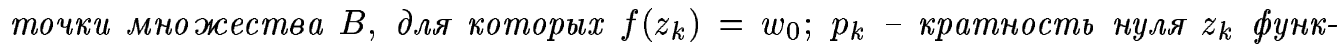
чии $f(z)-w_{0}$, то имеет место неравенство [1]

$$
g_{D}\left(w, w_{0}\right) \geqslant \sum_{k=0}^{\infty} p_{k} g_{B}\left(z, z_{k}\right)
$$

для всех $z \in B, w=f(z)$.

Вычитая из обеих частей неравенства (1) особенность в точке $z_{0}$ и переходя к пределу при $z \rightarrow z_{0}$, получим неравенство Митюка [2]

$$
\log r\left(D, w_{0}\right) \geqslant \log \left|a_{p}\right|+p \log r\left(B, z_{0}\right)+\sum_{k=1}^{\infty} p_{k} g_{B}\left(z_{0}, z_{k}\right)
$$

где $r(\Omega, a)$ - внутренний радиус области $\Omega$ относительно точки $a$ и

$$
f(z)-w_{0}=a_{p}\left(z-z_{0}\right)^{p}+o\left(\left(z-z_{0}\right)^{p}\right), \quad z \rightarrow z_{0}, \quad a_{p} \neq 0 .
$$

Работа выполнена при финансовой поддержке Российского фонда фундаментальных исследований, грант № 96-01-00007. 
Заметим, что неравенство (2) содержит известное неравенство Хеймана

$$
\left|a_{1}\right| \leqslant r\left(f(U), a_{0}\right)
$$

для регулярных в круге $U=\{z:|z|<1\}$ функций $f(z)=a_{0}+a_{1} z+\cdots$ и имеет многочисленные приложения в геометрической теории функций [3]. Пусть теперь $w_{l}, l=$ $1, \ldots, m,-$ различные точки области $f(B) ; p$ - фиксированное натуральное число; $z_{j l}$, $j=1, \ldots, p,-$ некоторые нули функции $f(z)-w_{l}$, причем каждьй нуль взят столько раз, какова его кратность $p_{j l}$ (в частности, мы предполагаем, что суммарная кратность $p$ выбранных точек не зависит от $l$ ). Пусть

$$
\begin{gathered}
f(z)-w_{l}=a_{j l}\left(z-z_{j l}\right)^{p_{j l}}+o\left(\left(z-z_{k l}\right)^{p_{j l}}\right), \quad z \rightarrow z_{j l}, \quad a_{j l} \neq 0, \\
j=1, \ldots, p, \quad l=1, \ldots, m,
\end{gathered}
$$

и $\delta_{l}, l=1, \ldots, m,-$ произвольные положительные числа. Суммируя по $i$ либо по $j$ неравенства вида (1) для всевозможных пар точек $w_{k}, w_{l}$ и $z_{i k}, z_{j l}, k \neq l$, умножая результат на $\delta_{k} \delta_{l}$ и вновь суммируя по $k$ и $l$, а затем повторяя аналогичную процедуру с неравенством (2) при $k=l$, получим симметричную оценку

$$
p \sum \delta_{k} \delta_{l} G\left(D, w_{k}, w_{l}\right) \geqslant \sum \delta_{k} \delta_{l} G\left(B, z_{i k}, z_{j l}\right)+\sum \delta_{l}^{2} \log \left|a_{j l}\right|
$$

(здесь и далее, если не оговорено противное, символы $\{\cdot\}, \sum$ и П означают соответственно совокупность, суммирование и произведение по всевозможным индексам, указанным в контексте, за исключением тех, при которых слагаемое в сумме равно $\infty$ либо неопределено, а сомножитель в произведении равен 0 либо $\infty$ ), где

$$
G(\Omega, z, \zeta)= \begin{cases}g_{\Omega}(z, \zeta), & \text { если } z \neq \zeta, \\ \log r(D, \zeta), & \text { если } z=\zeta\end{cases}
$$

Заметим, что линейная комбинация $\sum \delta_{k} \delta_{l} G\left(D, w_{k}, w_{l}\right)$ для любых $\delta_{l}$ уже встречалась в известных работах Шиффера [4] и Нехари [5]. Естественно возникает вопрос о справедливости неравенства (3) для произвольных вещественных $\delta_{l}$ (необязательно положительных). Конечно, в этом случае необходимо существенно использовать листность накрытия и, следовательно, можно ограничиться $p$-листными функциями [6, с. 469].

Следующий пункт носит вспомогательньй характер. Мы напоминаем понятие обобщенного приведенного модуля [7]-[9], в п. 3 доказываем неравенство (3) для произвольных $\delta_{l}$ и в конце рассматриваем некоторые приложения принципа мажорации (3) в геометрической теории функций комплексного переменного.

2. Приведенные модули. Пусть $B$ - открытое множество плоскости $\overline{\mathbb{C}}_{z}$, дополнение которого $E_{0}=\overline{\mathbb{C}}_{z} \backslash B$ имеет положительную гармоническую меру [6]. Пусть $z_{l}$, $l=1, \ldots, m,-$ различные точки множества $B ; \delta_{l}, l=1, \ldots, m,-$ произвольные действительные числа отличные от 0 и $\mu_{l}, \nu_{l}, l=1, \ldots, m,-$ произвольные положительные числа. Введем следующие обозначения: $Z=\left\{z_{l}\right\}, \Delta=\left\{\delta_{l}\right\}, \Psi=\left\{\psi_{l}\right\}, \psi_{l}=\psi_{l}(r) \equiv \mu_{l} r^{\nu_{l}}$, $l=1, \ldots, m$,

$$
E\left(z_{0}, r\right)=\left\{\begin{array}{lll}
\left\{z:\left|z-z_{0}\right| \leqslant r\right\} & \text { в случае конечной точки } z_{0}, & \\
\{z:|z| \geqslant 1 / r\} & \text { в случае } z_{0}=\infty,
\end{array} \quad r>0 .\right.
$$


При достаточно малом $r>0$ определим обобщенный конденсатор как упорядоченную совокупность

$$
C(r ; B, Z, \Delta, \Psi)=\left\{E_{0}, E\left(z_{1}, \psi_{1}(r)\right), \ldots, E\left(z_{m}, \psi_{m}(r)\right)\right\}
$$

с предписанными значениями $0, \delta_{1}, \ldots, \delta_{m}$ соответственно. По аналогии с обычньми конденсаторами модулем конденсатора $C(r ; B, Z, \Delta, \Psi)$ назовем величину

$$
|C(r ; B, Z, \Delta, \Psi)|=\sup \left\{\left(\iint_{\mathbb{C}_{z}}|\nabla v|^{2} d x d y\right)^{-1}\right\},
$$

где верхняя грань берется по всем вещественнозначным функциям $v(z)$, непрерывным в $\overline{\mathbb{C}}_{z}$, удовлетворяющим условию Липшища в некоторой окрестности каждой конечной точки, и таким, что $v(z)=0$ в окрестности множества $E_{0}$ и $v(z)=\delta_{l}$ на $E\left(z_{l}, \psi_{l}(r)\right), l=$ $1, \ldots, m$ (здесь $1 / 0=+\infty)$. Приведенным модулем мнохества $B$ относительно совокупностей $Z, \Delta, \Psi$ назовем предел

$$
M(B, Z, \Delta, \Psi)=\lim _{r \rightarrow 0}\left(|C(r ; B, Z, \Delta, \Psi)|+\frac{\nu}{2 \pi} \log r\right),
$$

где $\nu=\left(\sum \delta_{l}^{2} \nu_{l}^{-1}\right)^{-1}$. Непосредственно из определения приведенного модуля вытекает важное свойство монотонности: $M(B, Z, \Delta, \Psi) \leqslant M\left(B^{\prime}, Z, \Delta, \Psi\right)$ для любых $Z=\left\{z_{l}\right\}$, $z_{l} \in B \subset B^{\prime}, l=1, \ldots, m$, и любых $\Delta, \Psi$. Введем вспомогательные множества $\widetilde{E}\left(z_{l}, r\right)$, $l=1, \ldots, m$, как произвольные замкнутые множества в $\overline{\mathbb{C}}_{z}$, удовлетворяющие условию

$$
E\left(z_{l}, \psi_{l}\left(r_{1}\right)\right) \subset \widetilde{E}\left(z_{l}, r\right) \subset E\left(z_{l}, \psi_{l}\left(r_{2}\right)\right), \quad l=1, \ldots, m
$$

при некоторых положительных непрерьвных $r_{j}=r_{j}(r) \sim r, r \rightarrow 0,0<r<r_{0}, j=1,2$. Обобщенньй конденсатор, определенный как и в $(4)$, но с заменой $E\left(z_{l}, \psi_{l}(r)\right)$ на $\widetilde{E}\left(z_{l}, r\right)$, обозначим через $\widetilde{C}(r ; B, Z, \Delta, \Psi)$. Нетрудно убедиться в справедливости следующей леммы.

Лемма 1. Если при некоторых $\widetilde{E}\left(z_{l}, r\right), l=1, \ldots, m$, существует предел

$$
\lim _{r \rightarrow 0}\left(|\widetilde{C}(r ; B, Z, \Delta, \Psi)|+\frac{\nu}{2 \pi} \log r\right)
$$

то существует равный ему предел (5), и обратно, если существует предел (5), то существует равный ему предел (7) для любых $\widetilde{E}\left(z_{l}, r\right), l=1, \ldots, m$.

Для произвольной области $G \subset \overline{\mathbb{C}}_{z}$ обозначим через $g_{G}\left(z, z_{0}\right)$ функцию Грина этой области с полюсом в точке $z_{0} \in G$, доопределенную нулем во внешности $G$. Пусть $r\left(G, z_{0}\right)$ - внутренний радиус области $G$ относительно точки $z_{0}$. В случае $z_{0}=\infty$ полагаем

$$
r(G, \infty)=\exp \left(\lim _{z \rightarrow \infty}\left(g_{G}(z, \infty)-\log |z|\right)\right) .
$$


Лемма 2. Пусть множество $B$ и совокупности $Z, \Delta, \Psi$ такие же, как и выше, $u$ пусть $B_{l}$ означает связную компоненту мнохсества $B$, содержсащую точку $z_{l}$, $l=1, \ldots, m$ (при такой нумерации одна компонента может иметь несколько обозначений). Тогда приведенный модуль (5) существует и справедлива формула

$$
M(B, Z, \Delta, \Psi)=\frac{\nu^{2}}{2 \pi}\left(\sum \frac{\delta_{l}^{2}}{\nu_{l}^{2}} \log \frac{r\left(B_{l}, z_{l}\right)}{\mu_{l}}+\sum \frac{\delta_{l}}{\nu_{l}} \frac{\delta_{j}}{\nu_{j}} g_{B_{l}}\left(z_{j}, z_{l}\right)\right) .
$$

Доказательство леммы 2 аналогично соответствующим доказательствам в работах [7], [8]. Подробное исследование асимптотики конденсатора $C(r ; B, Z, \Delta, \Psi)$ приведено также в [9].

3. Основной результат. Функция $w=f(z)$, мероморфная в некоторой области $B \subset \overline{\mathbb{C}}_{z}$, назьвается $p$-листной в этой области, если она принимает в $B$ каждое комплексное значение $w$ не более чем в $p$ точках. Условимся говорить, что отображение $w=f(z)$ осуществляет полное $p$-кратное накрытие области $f(B)$ областью $B$, если каждой точке $w \in f(B)$ соответствует ровно $p$ прообразов в области $B$ с учетом их кратности и любая последовательность точек $z_{n} \in B$, стремящаяся к $\partial B$, переходит в последовательность точек, стремящуюся к $\partial f(B)$. Следующее утверждение естественно назвать приниипом мажсорации (ср. [6, гл. 8]).

Теорема 1. Пусть $B$ и $D$ - области, имеющие функиии Грина и расположснные в плоскостях $\overline{\mathbb{C}}_{z} u \overline{\mathbb{C}}_{w}$ соответственно, $w=f(z)$-мероморфная $p$-листная функиия в $B$, значения которой лехсат в $D$. Пусть $w_{l}, l=1, \ldots, m,-$ различные точки области $D$, каждая из которых имеет ровно р прообразов в $B$ с учетом их кратности, и пусть $z_{j l}, j=1, \ldots, p,-$ указанные прообразы точки $w_{l}, l=1, \ldots, m$ (каждый нуль функиии $f(z)-w_{l}$ получает столько обозначений какова его кратность). Тогда для любых действительных чисел $\delta_{l}, l=1, \ldots, m$, справедливо неравенство (3), где коэффициенты $a_{j l}$ определяются из разложсений

$$
\begin{gathered}
f(z)-w_{l}=a_{j l}\left(z-z_{j l}\right)^{p_{j l}}+o\left(\left(z-z_{j l}\right)^{p_{j l}}\right), \quad z \rightarrow z_{j l}, \quad a_{j l} \neq 0 \\
j=1, \ldots, p, \quad l=1, \ldots, m
\end{gathered}
$$

где в случае $z_{j l}=\infty\left(w_{l}=\infty\right)$ величину $z-z_{j l}\left(f(z)-w_{l}\right)$ необходимо заменить на $1 / z(1 / f(z)$ соответственно). Если, дополнительно, область $D$ имеет классическую функиию Грина, а отобрахсение $w=f(z)$ осуществляет полное р-кратное накрытие области $D$ областью $B$, то в неравенстве (3) достигается знак равенcmsa.

ДоКАЗАТЕЛЬСТВо. Можно считать, что все $\delta_{l}, l=1, \ldots, m$, отличны от 0 и область $B$ ограничена конечным числом аналитических жордановых кривых. Положим $W=\left\{w_{l}\right\}, \Delta=\left\{\delta_{l}\right\}, \Psi=\left\{t_{l}(r)\right\}, t_{l}(r) \equiv r, l=1, \ldots, m, \nu=\left(\sum \delta_{l}^{2}\right)^{-1}$. При достаточно малом $r>0$ в плоскости $\overline{\mathbb{C}}_{w}$ определен конденсатор $C(r ; D, W, \Delta, \Psi)$. По лемме 2 существует приведенньй модуль

$$
M(D, W, \Delta, \Psi)=\lim _{r \rightarrow 0}\left(|C(r ; D, W, \Delta, \Psi)|+\frac{\nu}{2 \pi} \log r\right) .
$$


Конденсатору $C(r ; D, W, \Delta, \Psi)$ в плоскости $\overline{\mathbb{C}}_{z}$ сопоставим конденсатор $\widetilde{C}(r ; B, \widetilde{Z}, \widetilde{\Delta}, \widetilde{\Psi})$, где $\widetilde{Z}=\left\{\widetilde{z}_{k l}\right\}, \widetilde{z}_{k l}, k=1, \ldots, n_{l}, l=1, \ldots, m,-$ нули функции $f(z)-w_{l}$ без учета кратности, $\widetilde{\Delta}=\left\{\delta_{k l}\right\}, \delta_{k l}=\delta_{l} / p, k=1, \ldots, n_{l} ; \widetilde{\Psi}=\left\{\psi_{k l}(r)\right\}, \psi_{k l}(r)=\left|\widetilde{a}_{k l}\right|^{-1 / p_{k l}} r^{1 / p_{k l}}$, $p_{k l}, k=1, \ldots, n_{l},-$ кратность точки $\widetilde{z}_{k l}, \sum_{k=1}^{n_{l}} p_{k l}=p, l=1, \ldots, m, \widetilde{a}_{k l}-$ коэффициент, соответствующий точке $\widetilde{z}_{k l} ; \widetilde{E}\left(\widetilde{z}_{k l}, r\right)$ - связная часть прообраза $E\left(w_{l}, r\right)$ при отображении $w=f(z)$, лежащая в окрестности точки $\widetilde{z}_{k l}$. Легко видеть, что множества $\widetilde{E}\left(\widetilde{z}_{k l}, r\right)$ удовлетворяют условиям вида (6). Ввиду лемм 1 и 2

$$
M(B, \widetilde{Z}, \widetilde{\Delta}, \widetilde{\Psi})=\lim _{r \rightarrow 0}\left(|\widetilde{C}(r ; B, \widetilde{Z}, \widetilde{\Delta}, \widetilde{\Psi})|+\frac{\widetilde{\nu}}{2 \pi} \log r\right)
$$

где

$$
\widetilde{\nu}=\left(\sum \delta_{k l}^{2} p_{k l}\right)^{-1}=p\left(\sum \delta_{l}^{2}\right)^{-1}=p \nu
$$

Пусть теперь $u(z)$ - потенциальная функция конденсатора $\widetilde{C}(r ; B, \widetilde{Z}, \widetilde{\Delta}, \widetilde{\Psi})$, т.е. вещественнозначная функция $u(z)$, непрерьвная в $\overline{\mathbb{C}}_{z}$, гармоническая в $B \backslash \bigcup_{k, l} \widetilde{E}\left(\widetilde{z}_{k l}, r\right)$, равная 0 на $\overline{\mathbb{C}}_{z} \backslash B$ и $\delta_{k l}$ на $\widetilde{E}\left(\widetilde{z}_{k l}, r\right)$. На множестве $f(B)$ определим функцию

$$
U(w)=\sum_{f(z)=w} u(z)
$$

Значение функции $U(w)$ для каждого $w$ состоит не более чем из $p$ слагаемых. Функция $U(w)$ непрерьвна в $\overline{f(B)}$, равна 0 на границе $f(B)$ и $\delta_{l}$ на $E\left(w_{l}, r\right)$ и удовлетворяет условию Липшица в некоторой окрестности каждой конечной точки $f(B)$ за исключением, быть может, конечного числа таких точек. Из принципа Дирихле, вьпуклости функции $y=x^{2}$ и конформной инвариантности интеграла Дирихле имеем

$$
|C(r ; D, W, \Delta, \Psi)|^{-1} \leqslant \iint_{f(B)}|\nabla U|^{2} d u d v \leqslant p \iint_{B}|\nabla u|^{2} d x d y .
$$

Учитьвая вновь принцип Дирихле, заключаем, что

$$
p|C(r ; D, W, \Delta, \Psi)| \geqslant|\widetilde{C}(r ; B, \widetilde{Z}, \widetilde{\Delta}, \widetilde{\Psi})| .
$$

В силу (8)-(10) имеем

$$
p M(D, W, \Delta, \Psi) \geqslant M(B, \widetilde{Z}, \widetilde{\Delta}, \widetilde{\Psi})
$$

или с учетом леммы 2

$$
\begin{aligned}
p \sum \delta_{l} \delta_{k} G\left(D, w_{k}, w_{l}\right) & \geqslant \sum \delta_{k} p_{i k} \delta_{l} p_{j l} G\left(B, \widetilde{z}_{i k}, \widetilde{z}_{j l}\right)+\sum \delta_{l}^{2} p_{k l} \log \left|\widetilde{a}_{k l}\right| \\
& =\sum \delta_{k} \delta_{l} G\left(B, z_{i k}, z_{j l}\right)+\sum \delta_{l}^{2} \log \left|a_{j l}\right|
\end{aligned}
$$

Неравенство (3) доказано.

Предположим, что область $D$ имеет классическую функцию Грина, а отображение $w=f(z)$ осуществляет полное $p$-кратное накрытие области $D$ областью $B$. Тогда существует потенциальная функция $\omega(w)$ конденсатора $C(r ; D, W, \Delta, \Psi)$. Сложная функция $\omega(f(z))$ является потенциальной для конденсатора $\widetilde{C}(r ; B, \widetilde{Z}, \widetilde{\Delta}, \widetilde{\Psi})$, причем ввиду 
конформной инвариантности интеграла Дирихле и $p$-кратности накрытия вьполняется равенство

$$
p|C(r ; D, W, \Delta, \Psi)|=|\widetilde{C}(r ; B, \widetilde{Z}, \widetilde{\Delta}, \widetilde{\Psi})| .
$$

С учетом (8) и (9) это дает знак равенства в (3). Теорема доказана.

Заметим, что в случае $p=1$ и однолистной функции $w=f(z)$ неравенство (3) превращается в равенство и является простым следствием инвариантности функции Грина при конформном отображении. При $p \geqslant 1$ и положительных $\delta_{l}$ это неравенство, как было показано вьше, справедливо для любых регулярных функций и вытекает из принципа Линделёфа и теоремы Митюка. В случае разных знаков у $\delta_{l}$ условие $p$-листности существенно. Это видно уже при $p=1$ из следующего примера. Пусть $B$ есть плоскость $\mathbb{C}_{z} \mathrm{c}$ разрезами вдоль лучей $[-\infty, 0]$ и $[1,+\infty]$ действительной оси и пусть $D=\mathbb{C}_{w} \backslash[1,+\infty]$. Обозначим через $w=f(z)$ ветвь степенной функции, заданную в $B$ соотношениями $w=r^{3 / 2} e^{i 3 \varphi / 2}, z=r e^{i \varphi},-\pi<\varphi<\pi$. Функция $w=f(z)$ двулистная, однако точкам $w_{1}=e^{3 \pi i / 8}$ и $w_{2}=e^{-3 \pi i / 8}$ соответствуют ровно по одному прообразу с учетом кратности: точки $z_{1}=e^{i \pi / 4}$ и $z_{2}=e^{-i \pi / 4}$ соответственно. Прямые вычисления показьвают, что

$$
\log r\left(D, w_{1}\right) r\left(D, w_{2}\right)-2 g_{D}\left(w_{1}, w_{2}\right)=2 \log \left(2 \sin \frac{3 \pi}{8}\right) .
$$

В то же время

$$
\log r\left(B, z_{1}\right) r\left(B, z_{2}\right)-2 g_{B}\left(z_{1}, z_{2},\right)+\log \left|f^{\prime}\left(z_{1}\right) f^{\prime}\left(z_{2}\right)\right|=2 \log \frac{3}{\sqrt{2}}
$$

что противоречит неравенству (3).

4. Приложения. Прежде всего отметим, что специальные свойства $p$-листных функций изучены в меньшей степени, чем те свойства, которые вьполняются для известных обобщений $p$-листных в среднем и др. Мы ограничимся здесь простейшими приложениями теоремы 1 в случае, когда область $B$ есть круг, $m=2$ и $\delta_{1}= \pm \delta_{2}=1$. Обозначим через $\boldsymbol{M}_{p}$ класс функций, мероморфных и $p$-листных в круге $U=\{z:|z|<1\}$. Точку $z_{0}$ круга $U$ назовем $n$-кратной точкой функиии $f(z)$, если она является нулем функции $f(z)-f\left(z_{0}\right)$ кратности $n$. Для заданной функции $f(z)$ класса $\boldsymbol{M}_{p}$ и точки $z_{0} \in U$ кратности $n$ определим постоянную $a\left(f, z_{0}\right)=f^{(n)}\left(z_{0}\right) / n$ !. Следующее утверждение можно рассматривать как дополнение в случае $p$-листных функций к известной лемме Шварца (ср. [6, с. 319, 323]).

ТЕОРема 2. Пусть функиия $f(z)$ принадлежсит классу $\boldsymbol{M}_{p} u|f(z)|<R$ для всех $z \in U$. Пусть $w u \omega-$ произвольные различные точки образа $f(U), p$-кратно покрываемые кругом $U$ при отображсении $f(z)$. Тогда для систем точек $\left\{z_{k}\right\}_{k=1}^{p}$, $\left\{\zeta_{k}\right\}_{k=1}^{p}$, переходящих в точки $w$ и $\omega$ соответственно и взятых с учетом кратности, справедливо неравенство

$$
\begin{aligned}
& \left(\frac{\left(R^{2}-|w|^{2}\right)\left(R^{2}-|\omega|^{2}\right)|w-\omega|^{2}}{\left|R^{2}-w \bar{\omega}\right|^{2}}\right)^{p} \\
& \quad \geqslant \prod\left|a\left(f, z_{k}\right) a\left(f, \zeta_{k}\right)\right| \frac{\prod\left|1-z_{k} \bar{z}_{l}\right| \cdot\left|1-\zeta_{k} \bar{\zeta}_{l}\right|}{\prod\left|z_{k}-z_{l}\right| \cdot\left|\zeta_{k}-\zeta_{l}\right|} \prod\left|\frac{z_{k}-\zeta_{l}}{1-z_{k} \bar{\zeta}_{l}}\right|^{2} .
\end{aligned}
$$

Знак равенства в (11) достигается для функиий, осуществляющих полное р-кратное накрытие круга $V=\{w:|w|<R\}$ кругом $U$. 
ДокаЗАТЕЛЬСтво. Обозначим $f(U)$ через $D$. По лемме 2 и из монотонности приведенного модуля

$$
\log r(V, w) r(V, \omega)-2 g_{V}(w, \omega) \geqslant \log r(D, w) r(D, \omega)-2 g_{D}(w, \omega)
$$

С другой стороны, согласно теореме 1 справедливо неравенство

$$
\begin{aligned}
p\left(\log r(D, w) r(D, \omega)-2 g_{D}(w, \omega)\right) & \geqslant \sum \log r\left(U, z_{k}\right) r\left(U, \zeta_{k}\right)+\sum G\left(U, z_{k}, z_{l}\right) \\
& +\sum G\left(U, \zeta_{k}, \zeta_{l}\right)-2 \sum g_{U}\left(z_{k}, \zeta_{l}\right)+\sum \log \left|a\left(f, z_{k}\right) a\left(f, \zeta_{k}\right)\right|
\end{aligned}
$$

с соответствуюшим утверждением о знаке равенства. Завершают доказательство простые вычисления внутренних радиусов и функций Грина для кругов $U$ и $V$. Теорема доказана.

Отметим два частных случая неравенства (11). Пусть $f(z) \in \boldsymbol{M}_{p},|f(z)|<R$ при $|z|<1$ и пусть в окрестности точки $z=0$ имеет место разложение $f(z)=a_{p} z^{p}+\cdots$, $a_{p} \neq 0$. Предположим, что для точки $w \in f(U)$ существует ровно $p$ прообразов с учетом кратности $z_{1}, \ldots, z_{p}$. Тогда из неравенства (11) имеем

$$
\left(\left(1-\left|\frac{w}{R}\right|^{2}\right)|w|^{2}\right)^{p} \geqslant\left|a_{p}\right|^{p} \prod\left|f^{\prime}\left(z_{k}\right)\right| \frac{\prod\left|1-z_{k} \bar{z}_{l}\right|}{\prod\left|z_{k}-z_{l}\right|} \prod\left|z_{k}\right|^{2 p}
$$

Если, дополнительно, точки $z_{1}, \ldots, z_{p}$ лежат на одной окружности $|z|=r<1$, то с учетом следствия 3 из [7] получим

$$
\left(1-\left|\frac{w}{R}\right|^{2}\right)|w|^{2} \geqslant\left|\frac{a_{p}}{p}\right|\left(1-r^{2 p}\right) r^{p+1} \sqrt[p]{\prod\left|f^{\prime}\left(z_{k}\right)\right|} .
$$

Знак равенства в обоих случаях достигается для функции $w=R z^{p}$ и точек $z_{k}=e^{2 \pi i k / p}$, $k=1, \ldots, p$.

Пусть теперь $z$ и $\zeta$ - две различные $p$-кратные точки функции $f(z)$ из теоремы 2 . В этом случае неравенство (11) имеет вид

$$
\begin{aligned}
& (p !)^{2} \frac{\left(R^{2}-|f(z)|^{2}\right)\left(R^{2}-|f(\zeta)|^{2}\right)|f(z)-f(\zeta)|^{2}}{\left|R^{2}-f(z) \overline{f(\zeta)}\right|^{2}} \\
& \quad \geqslant\left|f^{(p)}(z) f^{(p)}(\zeta)\right|\left(1-|z|^{2}\right)^{p}\left(1-|\zeta|^{2}\right)^{p}\left|\frac{z-\zeta}{1-\bar{z} \zeta}\right|^{2 p}
\end{aligned}
$$

Поскольку при полном $p$-кратном накрытии круга кругом возможна лишь одна $p$-кратная точка, последнее неравенство не точное. Применение симметризации относительно окружности [7] дает следующий результат. 
Теорема 3. Пусть функция $f(z)$ принадлехсит классу $\boldsymbol{M}_{p}, w u \omega-$ произвольные различные конечные точки образа $f(U), p$-кратно покрываемые кругом $U$ при отображснии $f(z)$. Пусть $\alpha_{f}(w, \omega)$ - линейная мера всех $\theta, 0 \leqslant \theta \leqslant 2 \pi$, для которых дуга окружности с кониами в $w, \omega$ и углом $\theta \kappa$ действительной оси в точке $w$ не принадлехит $f(U)$. Тогда для систем точек $\left\{z_{k}\right\}_{k=1}^{p},\left\{\zeta_{k}\right\}_{k=1}^{p}$, переходящих в точки $ш$ и $\omega$ соответственно и взятых с учетом кратности, справедливо неравенство

$$
\begin{aligned}
& |w-\omega|^{2 p} \sin ^{-4 p} \frac{\alpha_{f}(w, \omega)}{4} \\
& \quad \geqslant \prod\left|a\left(f, z_{k}\right) a\left(f, \zeta_{k}\right)\right| \frac{\prod\left|1-z_{k} \bar{z}_{l}\right| \cdot\left|1-\zeta_{k} \bar{\zeta}_{l}\right|}{\prod\left|z_{k}-z_{l}\right| \cdot\left|\zeta_{k}-\zeta_{l}\right|} \prod\left|\frac{1-z_{k} \bar{\zeta}_{l}}{z_{k}-\zeta_{l}}\right|^{2} .
\end{aligned}
$$

Знак равенства достигается, например, для функиии $f(z)=z^{p}+1 / z^{p}$ и точек $z_{k}=r e^{\pi i /(2 p)+2 \pi i k / p}, \zeta_{k}=r e^{-\pi i /(2 p)+2 \pi i k / p}, 0<r<1, k=1, \ldots, p$.

ДокАЗАТЕльство. Функция $\zeta=1 /(f(z)-\omega)$ принадлежит классу $\boldsymbol{M}_{p}$. Обозначим через $B$ образ круга $U$, возникающий при отображении этой функцией, $w_{0}=1 /(w-$ $\omega)$, и пусть $D=f(U)$. Ввиду конформной инвариантности функции Грина и согласно теореме 14 из [7] выполнено

$$
\begin{aligned}
\log r(D, w) r(D, \omega)+2 g_{D}(w, \omega) & =\log r\left(B, w_{0}\right) r(B, \infty)+2 g_{B}\left(w_{0}, \infty\right)+\log |w-\omega|^{2} \\
& \leqslant \log \left(|w-\omega|^{2} \sin ^{-4} \frac{\alpha_{f}(w, \omega)}{4}\right) .
\end{aligned}
$$

Оценка левой части неравенства снизу осуществляется с помощью теоремы 1 так же, как и при доказательстве теоремы 2. Для проверки случая равенства удобнее всего воспользоваться утверждениями о знаке равенства в теореме 1 и в теореме 14 из [7]. Теорема доказана.

СлЕДСТВИЕ. Пусть функиия $f(z) \in \boldsymbol{M}_{p}$ и пусть в окрестности точки $z=0$ имеет место разложсние $f(z)=z^{p}+\cdots$. Предположим, что для точки $w \in$ $f(U)$ имеется ровно $p$ прообразов с учетом кратности $z_{1}, \ldots, z_{p}$ и любая дуга окружности, соединяющая точки 0 и $w$, не принадлежит $f(U)$. Тогда имеет место оиенка

$$
|w|^{2 p} \geqslant \prod\left|a\left(f, z_{k}\right)\right| \frac{\prod\left|1-z_{k} \bar{z}_{l}\right|}{\prod\left|z_{k}-z_{l}\right|} \prod\left|z_{k}\right|^{-2 p} .
$$

Для функций $f(z)$ класса $\boldsymbol{M}_{p}$ введем обозначение

$$
\mu_{f}(w, \omega)=\int_{\sigma} t^{-1}(1-t)^{-1} d t, \quad \sigma=\{t \in(0,1): w+t(\omega-w) \notin f(U)\} .
$$

ТЕОремА 4. Если функиия $f(z)$ принадлежсит классу $\boldsymbol{M}_{p}$ и для данных различных конечных точек $w, \omega \in f(U)$ имеется ровно по $p$ прообразов с учетом кратности $z_{1}, \ldots, z_{p}$ и $\zeta_{1}, \ldots, \zeta_{p}$ соответственно, то

$$
\operatorname{ch}^{-4 p} \frac{\mu_{f}(w, \omega)}{4}|w-\omega|^{2 p} \geqslant \prod\left|a\left(f, z_{k}\right) a\left(f, \zeta_{k}\right)\right| \frac{\prod\left|1-z_{k} \bar{z}_{l}\right| \cdot\left|1-\zeta_{k} \bar{\zeta}_{l}\right|}{\prod\left|z_{k}-z_{l}\right| \cdot\left|\zeta_{k}-\zeta_{l}\right|} \prod\left|\frac{z_{k}-\zeta_{l}}{1-z_{k} \bar{\zeta}_{l}}\right|^{2} .
$$

Знак равенства достигается для функиии $f(z)=z^{p}+1 / z^{p}$ и точек $z_{k}=r e^{2 \pi i k / p}$, $\zeta_{k}=r e^{\pi i / p+2 \pi i k / p}, 0<r<1, k=1, \ldots, p$. 
ДокАЗАТЕЛЬСтво. Обозначим через $B$ плоскость $\overline{\mathbb{C}}_{w}$, разрезанную по отрезку $\{w+t(\omega-w):(1-\tau) / 2 \leqslant t \leqslant(1+\tau) / 2\}$, где $\tau=\operatorname{th}\left(\mu_{f}(w, \omega) / 4\right)$, и пусть $D=f(U)$. По теореме 2 из [8]

$\log r(D, w) r(D, \omega)-2 g_{D}(w, \omega) \leqslant \log r(B, w) r(B, \omega)-2 g_{B}(w, \omega)=2 \log \left(1-\tau^{2}\right)|w-\omega|$.

Осталось воспользоваться теоремой 1. Случай равенства проверяется непосредственно. Теорема доказана.

Как показывают доказательства теорем 3 и 4, принцип мажорации для $p$-листных функций позволяет распространить и уточнить результаты, полученные ранее методом симметризации для регулярных либо регулярных и однолистных функций, на случай $p$-листных отображений.

\section{СПИСОК ЦИТИРОВАННОЙ ЛИТЕРАТУРЫ}

[1] Стоилов С. Теория функций комплексного переменного. Т. 2. М.: ИЛ, 1962.

[2] Митюк И.П. Принцип симметризации для многосвязных областей // Докл. АН СССР. 1964. T. 157. № 2. C. 268-270.

[3] Митюк И. П. Симметризационные методы и их применение в геометрической теории функций. Введение в симметризационные методы. Краснодар: Кубанский гос. ун-т, 1980.

[4] Шиффер М. Некоторые новые результаты в теории конформных отображений // Приложение к книге Р. Куранта "Принцип Дирихле, конформные отображения и минимальные поверхности". М.: ИЛ, 1953.

[5] Nehari Z. Some inequalities in the theory of functions // Trans. Amer. Math. Soc. 1953. V. 75. №2. P. 256-286.

[6] Голузин Г. М. Геометрическая теория функций комплексного переменного. М.: Наука, 1966.

[7] Дубинин В.Н. Некоторые свойства внутреннего приведенного модуля // Сиб. матем. ж. 1994. Т. 35. № 4. С. 774-792.

[8] Дубинин В.Н. Симметризация, функция Грина и конформные отображения // Аналитическая теория чисел и теория функций. Вьп. 13. Зап. научных семин. ПОМИ. 1996. Т. 226. C. $80-92$.

[9] Дубинин В. Н. Асимптотика модуля вырождающегося конденсатора и некоторые ее применения // Аналитическая теория чисел и теория функций. Вып. 14. Зап. научных семин. ПОМИ. 1997. T. 237. C. $56-73$.

Институт прикладной математики ДВО РАН, г. Владивосток

Поступило

E-mail: dubn@ipm.marine.su

08.01 .97 